\title{
Two distinct patterns of treatment resistance: clinical predictors of treatment resistance in first-episode schizophrenia spectrum psychoses
}

\author{
J. Lally ${ }^{1,2,3 *}$, , O. Ajnakina ${ }^{1}$ t, M. Di Forti ${ }^{4}$, A. Trotta ${ }^{1}$, A. Demjaha ${ }^{1}$, A. Kolliakou ${ }^{6}$, V. Mondelli ${ }^{5,6}$, \\ T. Reis Marques ${ }^{1}$, C. Pariante ${ }^{5,6}$, P. Dazzan ${ }^{1,5}$, S. S. Shergil ${ }^{1,2,5}$, O. D. Howes ${ }^{1,7}$, A. S. David ${ }^{1,5}$, \\ J. H. MacCabe ${ }^{1,2}$, F. Gaughran ${ }^{1,2} \ddagger$ and R. M. Murray ${ }^{1,2} \ddagger$ \\ ${ }^{1}$ Department of Psychosis Studies, Institute of Psychiatry, Psychology E Neuroscience (IoPPN), King's College London, London, UK \\ ${ }^{2}$ National Psychosis Service, South London and Maudsley NHS Foundation Trust, London, UK \\ ${ }^{3}$ Department of Psychiatry, Royal College of Surgeons in Ireland, Beaumont Hospital, Dublin, Ireland \\ ${ }^{4}$ MRC Social, Genetic \& Developmental Psychiatry Centre, Institute of Psychiatry, Psychology \& Neuroscience, King's College London, London, UK \\ ${ }^{5}$ National Institute for Health Research (NIHR) Mental Health Biomedical Research Centre at South London and Maudsley NHS Foundation Trust \\ and King's College London, UK \\ ${ }^{6}$ Department of Psychological Medicine, Institute of Psychiatry, Psychology and Neuroscience, Kings College London, UK \\ ${ }^{7}$ MRC Clinical Sciences Centre (Imperial Hammersmith Campus)
}

Background. Clozapine remains the only evidence-based antipsychotic for treatment-resistant schizophrenia (TRS). The ability to predict which patients with their first onset of schizophrenia would subsequently meet criteria for treatment resistance (TR) could help to diminish the severe functional disability which may ensue if TR is not recognized and correctly treated.

Method. This is a 5-year longitudinal assessment of clinical outcomes in a cohort of 246 first-episode schizophrenia spectrum patients recruited as part of the NIHR Genetics and Psychosis (GAP) study conducted in South London from 2005 to 2010. We examined the relationship between baseline demographic and clinical measures and the emergence of TR. TR status was determined from a review of electronic case records. We assessed for associations with early-, and late-onset TR, and non-TR, and differences between those TR patients treated with clozapine and those who were not.

Results. Seventy per cent $(n=56)$ of TR patients, and $23 \%$ of the total study population $(n=246)$ were treatment resistant from illness onset. Those who met criteria for TR during the first 5 years of illness were more likely to have an early age of first contact for psychosis (<20 years) [odds ratio (OR) 2.49, 95\% confidence interval (CI) 1.25-4.94] compared to those with non-TR. The relationship between an early age of first contact ( $<20$ years) and TR was significant in patients of Black ethnicity (OR 3.71, 95\% CI 1.44-9.56); and patients of male gender (OR 3.13 95\% CI 1.35-7.23).

Conclusions. For the majority of the TR group, antipsychotic TR is present from illness onset, necessitating increased consideration for the earlier use of clozapine.

Received 24 March 2016; Revised 13 July 2016; Accepted 19 July 2016; First published online 8 September 2016

Key words: Clozapine, first-episode psychosis, longitudinal, schizophrenia, treatment-resistant.

\section{Introduction}

Treatment-resistant schizophrenia (TRS) is a major cause of disability and functional impairment and affects up to $30 \%$ of those diagnosed with schizophrenia (Meltzer, 1997). Clozapine is the only evidencedbased effective medication for TRS. In the UK, it is recommended that clozapine be offered to people with schizophrenia whose illness has not responded

* Address for correspondence: Dr J. Lally, Department of Psychosis Studies, Institute of Psychiatry, Psychology and Neuroscience (IoPPN), King's College London, London, UK.

(Email: john.lally@kcl.ac.uk)

+ Both are first named authors and should be acknowledged as such.

$\ddagger$ Both are last named authors and should be acknowledged as such. adequately to treatment despite the sequential use of adequate doses of at least two different antipsychotic drugs (NICE, 2014). At present it is not possible to predict those who will not respond to first-line antipsychotic treatments.

Early identification of patients who require clozapine has the potential to improve clinical outcomes and minimize the social and functional disability that results from prolonged psychosis (Lewis et al. 2006; Wheeler et al. 2009; Stroup et al. 2016). Previous studies investigating risk factors for treatment resistance (TR) have utilized clozapine prescription rates as a proxy measure of TR (Nielsen et al. 2012b; Stroup et al. 2014; Schneider et al. 2015); however, given the under-utilization of clozapine in clinical practice, such studies do not measure all people with TR. 
Although, a few potential risk factors for TR, such as poor premorbid functioning, living in less urban areas, co-morbid personality disorder, longer duration of untreated psychosis (DUP), greater severity of negative symptoms, and a younger age of illness onset have been suggested (Vanelle et al. 1994; Meltzer, 1997; Schennach et al. 2012; Ortiz et al. 2013; Frank et al. 2015; Martin \& Mowry, 2016; Wimberley et al. 2016), the predictive value of specific clinical and demographic factors on TR in first-episode schizophrenia has not yet been widely investigated (Lin et al. 2008). Further, while there is a large literature investigating predictors of treatment response and remission from illness onset (Menezes et al. 2006; Carbon \& Correll, 2014), TR has not been examined longitudinally as an outcome measure in first-episode psychosis (FEP).

Therefore in our study, using a quasi-prospective cohort design, we sought to identify clinical and demographic risk factors, noted at first contact for psychosis, which were predictive of TR as assessed at the end of the first 5 years of illness. We explored associations between TR and a number of risk predictors, including type and severity of symptoms, gender, age at first contact for psychosis, ethnicity, DUP and premorbid level of functioning. We looked for differential clinical and social factors between those who showed 'early-resistant' TR (E-TR) (within 6 months of first onset of schizophrenia with no period of remission from illness onset) and those with 'late-resistant' TR (L-TR). We further assessed for differences between those with E-TR and those who did not meet criteria for treatment resistance (non-TR), and those with L-TR and those who were non-TR. We additionally compared the TR patients treated with clozapine with those who met the criteria for TR but had not received clozapine, in terms of sociodemographic and clinical characteristics at the time of first illness onset.

\section{Method}

\section{Sample ascertainment}

Participants were recruited as part of the National Institute of Health Research (NIHR) Biomedical Research Centre (BRC) Genetics and Psychosis (GAP) study conducted in the urban centre of South London, UK. Further details of the study are available in Di Forti et al. (2014). For the purpose of the present study, only FEP cases who met criteria for the following ICD-10 diagnoses: F20.0, F25.0, F28.0, F29.0 (referred to as schizophrenia spectrum psychosis; there are no cases of schizotypal disorder included) (WHO, 1992) validated by administration of the Schedules for Clinical Assessment in Neuropsychiatry (SCAN; WHO, 1994) were included at baseline. All cases had been admitted to psychiatric inpatient units or seen by community-based mental health teams of the South London and Maudsley (SLaM) NHS Foundation Trust, London between December 2005 and October 2010. All recruited cases were aged 1865 years and were resident in the study catchment area. The study exclusion criteria were (1) evidence of psychotic symptoms precipitated by an organic cause; (2) evidence of transient psychotic symptoms resulting from acute intoxication as defined by ICD10; (3) moderate or severe learning disabilities as defined by ICD-10; or (4) head injury causing clinically significant loss of consciousness.

\section{Ethics}

The BRC-GAP study was granted ethical approval by the South London and Maudsley and Institute of Psychiatry Local Research Ethics Committee (reference no. 05/Q0706/158). All cases gave informed written consent after reading a detailed information sheet.

\section{Data ascertainment at baseline}

\section{Sociodemographic characteristics}

Information on sociodemographic characteristics was collated using the Medical Research Council (MRC) Socio-demographic Schedule modified version (Mallett et al. 2002). Age at first contact with mental health services for psychosis was further categorized into four groups based on the results of interquartile analyses (18-20, 21-25, 26-30, >31 years). Ethnicity was selfascribed using the 16 categories employed by the UK Census in 2001 (http://www.statistics.gov.uk/census 2001). A detailed assessment of lifetime patterns of cannabis use and alcohol intake was collated employing the Cannabis Experience Questionnaire modified version (Di Forti et al. 2009).

\section{Clinical assessments}

The baseline diagnoses were made from face-to-face interviews and mental health records according to ICD-10 criteria (WHO, 1992) utilizing the Operational Criteria Checklists (OPCRIT; McGuffin et al. 1991). The OPCRIT system consists of a 90-item checklist and uses computerized diagnostic algorithms based on published criteria to provide a diagnostic category for each subject employing a number of classification systems (McGuffin et al. 1991). The degree of psychopathology at first presentation to mental health services was measured on the Positive and Negative Syndrome Scale (PANSS; Kay et al. 1987). Overall functional disability was assessed using the Global Assessment of Functioning (GAF). DUP was defined as the difference between the date of onset of psychotic 
symptoms to the date of treatment with antipsychotic medications (Singh et al. 2005; Malla et al. 2006).

The Family Interview for Genetic Studies (FIGS; https://www.nimhgenetics.org/interviews/figs) and clinical records were used to obtain information about patients' family history of mental health problems. A family history of psychosis variable was derived following consensus diagnoses based on the available information and referred to the presence or absence of a current or past psychotic disorder in at least one first-degree relative.

Current IQ was estimated using Wechsler Adult Intelligence Scale, version III (WAIS-III; Wechsler, 1997) and pre-morbid IQ was estimated using the Wechsler Test of Adult Reading (WTAR; Wechsler, 2001). Premorbid and current IQ estimates, and the difference in IQ estimate between the premorbid and current IQ were compared between the TR and non-TR groups.

\section{Tracing patients at follow-up}

Approximately 5 years after first contact for psychosis, a retrospective database search of clinical history was carried out using the Electronic Psychiatric Clinical Records (EPCRs) database in the SLaM. The EPCRs are the primary clinical records system within the Trust. The EPCRs were searched from the time of FEP for a follow-up period of 5 years in order to complete the data extraction. All deaths and emigrations up to and including those that occurred during the final year of follow-up were identified by a case-tracing procedure with the Office for National Statistics (ONS) for England and Wales and the General Register Office (GRO) for Scotland.

\section{Data at follow-up}

At follow-up, extensive information was collated across clinical and social outcome domains, from clinical records using the WHO Life Chart Schedule (LCS) extended version (Sartorius et al. 1996). The LCS is designed to provide retrospective assessments of the long-term course of schizophrenia. Detailed information was collated on medication history including the number of antipsychotic medications used prior to commencing clozapine, medication initiation/discontinuation dates, antipsychotic dose, medication adherence and the primary reason for changing or discontinuing each antipsychotic medication.

\section{Definitions of TR}

Patients were defined as having TR if they had been treated with clozapine at any point during the 5-year follow-up period. We also defined cases as TR if during the follow-up period they showed little or no symptomatic improvement to two consecutive treatments with antipsychotic medications of adequate dose and duration (at least 6 weeks), even though they were not commenced on clozapine (NICE, 2014). An adequate daily dose of antipsychotic medication was defined according to a daily dose of at least 400 mg chlorpromazine equivalence (Leucht et al. 2014). We only included as cases those patients, who failed to respond, and not those who were intolerant of antipsychotic medications or those who self-discontinued medication.

Those who met the criteria for TR were divided into two subgroups: (1) E-TR were those cases who met criteria for TR and who did not experience a symptomatic remission from the time of the first presentation and (2) L-TR were those cases who had experienced a response to antipsychotics and attained a symptomatic remission (of at least 6 months duration), but at a later stage failed to respond to the ongoing use of nonclozapine antipsychotics, meeting the criteria for TR.

Similar to earlier work conducted in the same geographical region (Morgan et al. 2014; Revier et al. 2015), and in keeping with work from Andreasen et al. (2005), using information extracted from clinical records, remission was defined as the absence of overt psychotic symptoms, including positive or and negative symptoms (operationalized as a score of 0 or 1 on rating scale 2 in the SCAN (WHO, 1994); $0=a b-$ sence, 1 = symptom occurred, but fleeting, 2 = symptom definitely present, $3=$ symptom present more or less continuously) for $\geqslant 6$ months. This definition of remission was not dependent on the presence of non-psychotic symptoms (e.g. depressed mood), nor whether the patients were receiving treatment during remission.

\section{Data analysis}

Comparisons between the groups were conducted using $\chi^{2}$ tests or Fisher's exact test for categorical variables and $t$ test or Mann-Whitney $U$ test for continuous variables. Penalized logistic regression (PLR) was used to analyse the relationship between the predictors for TR (Heinze \& Schemper, 2002). To explore the moderating effects of gender and ethnicity in predicting the risk for TR, we examined whether there were significant interactions between these variables and the independent predictors of TR status at follow-up. Confounding factors were identified through correlation analyses between the primary outcome (i.e. TR v. non-TR at 5 years) and a number of clinical and sociodemographic factors measured at baseline and during the follow-up period. All variables with $p<$ 0.05 were included in the final model as confounding factors. Consequently, we adjusted the PLR analyses for the following variables: age at first contact for 
psychosis; living arrangements, and employment status and alcohol/substance misuse during the followup period. All analyses were conducted in Stata release 14 (StataCorp LP, USA).

\section{Results}

\section{Sample characteristics}

At first onset of psychosis, the sample comprised 283 cases with a diagnosis of a schizophrenia spectrum psychosis (ICD-10 classification codes: F20.0, F25.0, F28.0, F29.0), with an average age of 27.7 (S.D. = 8.6) years. Seventy three per cent were admitted to hospital at the time of their first presentation with psychosis and at the time of study recruitment, with $27 \%$ recruited from community based mental health settings. There were no significant differences between those recruited from an inpatient setting and those from a community setting in mean total PANSS scores $(p=0.35)$, and mean negative PANSS scores $(p=0.70)$ between those recruited from an inpatient setting and those from a community setting. Inpatients had significantly increased mean positive PANSS scores compared to those recruited from community settings $(p=0.02)$.

A flow chart depicting how the cases were traced and administrative outcomes is presented in Fig. 1. Approximately 5 years (mean $=5.4$, s.D. $=2.5$ years; 1310 persons-years) after first contact for psychosis, $10(3.5 \%)$ cases had emigrated, four $(1.4 \%)$ had died, two $(0.7 \%)$ were excluded on the basis of lack of sufficient information to trace these patients over time and $21(7.4 \%)$ cases were unable to be contacted. Those who died were significantly older (Supplementary Table S1).

\section{Core analytic cohort}

We successfully traced 246 (86.7\%) of the original cohort. Of these, $67.5 \%$ were male and $60.0 \%$ were of Black ethnicity. There were 240 cases with sufficient treatment information over the follow-up period to determine TR or non-TR status. Eighty-one (33.7\%) of the cases met our criteria for TR and 159 (66.3\%) were nonTR. The other six ( $2.4 \%$ of the core analytic cohort) cases had not received an adequate trial of antipsychotic to allow it to be determined if criteria for TR, or for non-TR was met.

Comparisons in baseline sociodemographic and clinical characteristics between the TR group and non-TR group are presented in Table 1 . The TR group had a significantly younger age at first contact for FEP (mean $=25.2$, S.D. $=6.4$ years) than the non-TR group (mean $=27.9$, S.D. $=8.3$ years $)\left(t_{238}=2.57, p=0.01\right)$. The median length of DUP for the non-TR group was 4 weeks [interquartile range $(\mathrm{IQR})=1-17$ ] which was not significantly different from DUP in the TR group (median $=4.5$ weeks, IQR =1-26; Mann-Whitney $U$ test $=-0.92, p=0.36$ ). Patients with TR did not have an increased family history of psychosis compared to patients in the non-TR group $\left(\chi^{2}=0.10, \mathrm{df}=1, p=\right.$ $0.75)$. There was no significant difference between the mean IQ in the TR group (mean =92.6, S.D. $=13.0)$ compared to the non-TR group (mean $=87.5$, S.D. $=16.2$; Mann-Whitney $U$ test $=-1.25, p=0.22$ ). The mean premorbid IQ was higher in the TR group (mean=92.8, S.D. =9.6) compared to those in the non-TR group $($ mean $=88.5$, s.D. $=11.0)$, but this did not reach statistical significance (Mann-Whitney $U$ test $=-1.58, p=0.11$ ). There were no other significant differences between the groups in terms of other baseline socio-demographic characteristics.

\section{Predictors of TR}

Baseline clinical predictors of TR at 5-year follow up for the whole sample and stratified by ethnicity and gender are presented in Table 2. Those patients who were defined as TR were more likely to have an early age of first contact with mental health services $(<20$ years) compared to the non-TR group [odds ratio (OR) 2.49, 95\% confidence interval (CI) 1.25-4.94]. There were significant interactions between age at first contact for psychosis with ethnicity $(p=0.02)$ and gender $(p=0.002)$. Therefore, in addition to exploring the impact of age at first contact in predicting TR in the cohort, we further stratified the analyses by gender and ethnicity. We observed that this relationship between an early age of first contact for psychosis $(<20$ year) and TR was significant in cases of Black ethnicity (OR 3.71, 95\% CI 1.44-9.56) and in male patients (OR 3.13, 95\% CI 1.35-7.23) (Table 3).

\section{'Clozapine' group v. 'met criteria' group}

Among those patients who were defined as TR, 38 cases $(46.9 \%$ of $n=81$, and $15.4 \%$ of 246 cases with a diagnosis of schizophrenia spectrum psychosis at study recruitment) commenced treatment with clozapine; whereas 43 cases $(53.1 \%$ of $n=81)$ met criteria for clozapine treatment but did not commence it. A higher proportion of patients in the clozapine group $(91 \%)$ lived with family members or friends compared to the 'met criteria' group $(48.0 \%)\left(\chi^{2}=6.04, p=0.01\right)$. The clozapine group exhibited more severe psychopathology $\left(t_{27}=2.49, p=0.02\right)$ and negative symptoms (Mann-Whitney $U$ test $=2.410, p=0.03$ ) compared to the 'met criteria' group at first contact for psychosis (Supplementary Table S2).

The clozapine group had increased negative symptoms (mean 21.7, S.D. $=9.1$ ) compared to the non-TR 


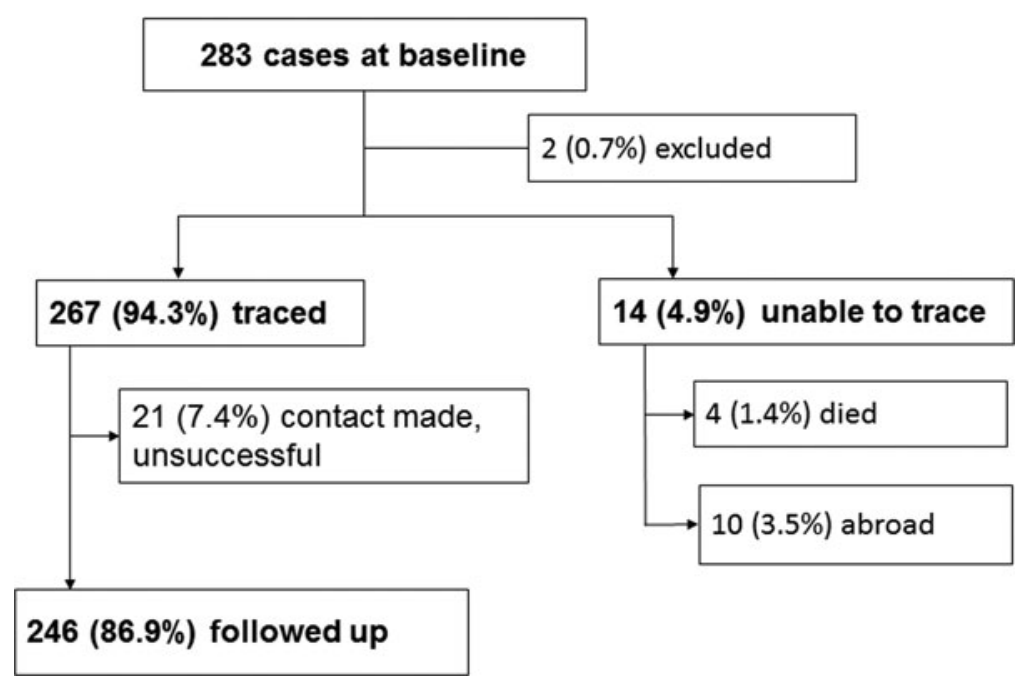

Fig. 1. Flow chart of traced cases and administrative outcomes.

Table 1. Baseline sample characteristics for the non-treatment-resistance (TR) and TR groups

\begin{tabular}{|c|c|c|c|c|c|}
\hline \multirow[b]{2}{*}{ Characteristic } & \multirow{2}{*}{$\begin{array}{l}\text { Non-TR }(N=159)(66.3 \%) \\
\text { Mean (s.D. }) / n(\%)\end{array}$} & \multirow{2}{*}{$\begin{array}{l}\text { TR }(N=81)(33.7 \%) \\
\text { Mean (s.D.) } / n(\%)\end{array}$} & \multicolumn{3}{|c|}{ Test statistics } \\
\hline & & & $t / U / \chi^{2}$ & $\mathrm{df}$ & $p$ \\
\hline DUP, weeks, median (IQR) & $4(1-17)$ & $4.5(1-26)$ & -0.92 & & 0.36 \\
\hline Age, years & $27.9(8.3)$ & $25.2(6.4)$ & 2.57 & 238 & 0.01 \\
\hline \multicolumn{6}{|l|}{ Gender } \\
\hline Female & $52(32.7)$ & $27(33.3)$ & 0.01 & 1 & 0.92 \\
\hline Male & $107(67.3)$ & $54(66.7)$ & & & \\
\hline \multicolumn{6}{|l|}{ Ethnicity } \\
\hline White ethnic groups & $48(36.6)$ & $30(44.1)$ & 1.05 & 1 & 0.36 \\
\hline Black ethnic groups & $83(63.4)$ & $38(55.9)$ & & & \\
\hline \multicolumn{6}{|l|}{ Living arrangements } \\
\hline Alone & $41(42.3)$ & $16(40.0)$ & 0.06 & 1 & 0.85 \\
\hline Not alone & $56(57.7)$ & $24(60.0)$ & & & \\
\hline \multicolumn{6}{|l|}{ Relationship status } \\
\hline Single/separated & $78(80.4)$ & $33(80.5)$ & 0.00 & 1 & 0.99 \\
\hline Stable relationship & $19(19.6)$ & $8(19.5)$ & & & \\
\hline \multicolumn{6}{|l|}{ Cannabis use } \\
\hline None/infrequent & $75(67.6)$ & $32(66.7)$ & 0.01 & 1 & 0.91 \\
\hline Every day & $36(32.4)$ & $16(33.3)$ & & & \\
\hline \multicolumn{6}{|l|}{ Alcohol intake } \\
\hline 0-14 units/week & $83(86.5)$ & $52(89.7)$ & 0.34 & 1 & 0.62 \\
\hline$>15$ units/week & $13(13.5)$ & $6(10.3)$ & & & \\
\hline IQ minus premorbid IQ & $1.01(1.5)$ & $0.1(2.81)$ & 0.30 & 67 & 0.76 \\
\hline
\end{tabular}

S.D., Standard deviation; df, degrees of freedom; IQR, interquartile range; DUP, duration of untreated psychosis.

group (mean 15.4, S.D. =6.0; Mann-Whitney $U$ test $=$ $-2.22 p=0.02)$.

\section{E-TR v. L-TR and v. the non-TR groups}

Among those with TR, 56 (70.0\% of 80 with TR) were E-TR and $24(30.0 \%$ of $n=80)$ were L-TR. The E-TR group expressed more severe psychopathology at baseline (mean total PANSS score $=69.9$, s.D. $=20.4$ ) than the
non-TR group (mean total PANSS score $=60.9$ s.D. $=16.4$; $\left.t_{95}=-1.98, p=0.05\right)$ (Supplementary Table S3). Cases in the L-TR group were significantly younger at the time of first contact for psychosis (mean $=23.7$, s.D. $=5.1$ years) compared to the non-TR group ( mean $=27.9$, s.D. $=8.3$ years; $t_{181}=2.44, p=0.02$ ) (Supplementary Table S4).

A higher proportion of the E-TR group (74\% of $n=56)$ were male compared to the L-TR group $(46 \%)\left(\chi^{2}=6.39\right.$, 
Table 2. Baseline clinical predictors of treatment resistance (TR) in a sample with schizophrenia spectrum disorder

Total sample $(N=246)$

\begin{tabular}{|c|c|c|}
\hline & Non-TR & $\begin{array}{l}\text { TR } \\
\text { OR }(95 \% \mathrm{CI})\end{array}$ \\
\hline & & \\
\hline \multicolumn{3}{|l|}{ Age group ${ }^{a}$} \\
\hline$>31$ years & - & $0.55(0.27-1.12)$ \\
\hline 26-30 years & - & $1.14(0.57-2.28)$ \\
\hline $21-25$ years & - & $0.70(0.37-1.32)$ \\
\hline$<20$ years & - & $2.49^{* * *}(1.25-4.94)$ \\
\hline \multicolumn{3}{|l|}{ Psychopathology } \\
\hline PANSS Total & - & $1.01(0.98-1.04)$ \\
\hline PANSS Positive & - & $1.01(0.94-1.07)$ \\
\hline PANSS Negative & - & $1.03(0.96-1.11)$ \\
\hline GAF Disability & - & $0.98(0.96-1.01)$ \\
\hline GAF Symptoms & & $0.97(0.94-1.00)$ \\
\hline $\begin{array}{l}\text { PANSS Lack of } \\
\text { judgement and insight }\end{array}$ & - & $1.14(0.89-1.47)$ \\
\hline $\begin{array}{l}\text { PANSS Conceptual } \\
\text { disorganization }\end{array}$ & & $1.13(0.83-1.56)$ \\
\hline
\end{tabular}

OR, odds ratio; CI, confidence interval; S.D., standard deviation; df, degrees of freedom; PANSS, Positive and Negative Syndrome Scale, GAF, Global Assessment of Functioning Scale; DUP, duration of untreated psychosis.

${ }^{a}$ Adjusted for alcohol use, illicit substance use and living arrangements at follow-up.

${ }^{\mathrm{b}}$ Adjusted for age at first contact, alcohol use, illicit substance use and living arrangements at follow-up. ${ }^{*} p<0.05,{ }^{* *} p<0.01,{ }^{* * *} p<0.001$.

$p=0.01)$. There were no other significant differences between the groups in terms of ethnicity, sociodemographic and clinical characteristics at baseline.

\section{Discussion}

This is the largest study of a first-episode schizophrenia spectrum psychosis cohort to assess the clinical and demographic risk factors associated with the emergence of TR. TR illness was seen in $34 \%$ of patients by 5 years follow-up. Our principal finding was that $70 \%$ of the TR cases did not respond to antipsychotics from illness onset.

An earlier age of first contact for psychosis was strongly associated with TR at 5 years follow-up. This finding of an association between a first contact with mental health services for psychosis before the age of 20 and TR was most marked for males, and for those of Black ethnicity. Early-onset schizophrenia is associated with worse outcomes compared to adult-onset schizophrenia (Ropcke \& Eggers, 2005; Vyas et al. 2007), and a younger age of illness onset has been previously associated with the emergence of

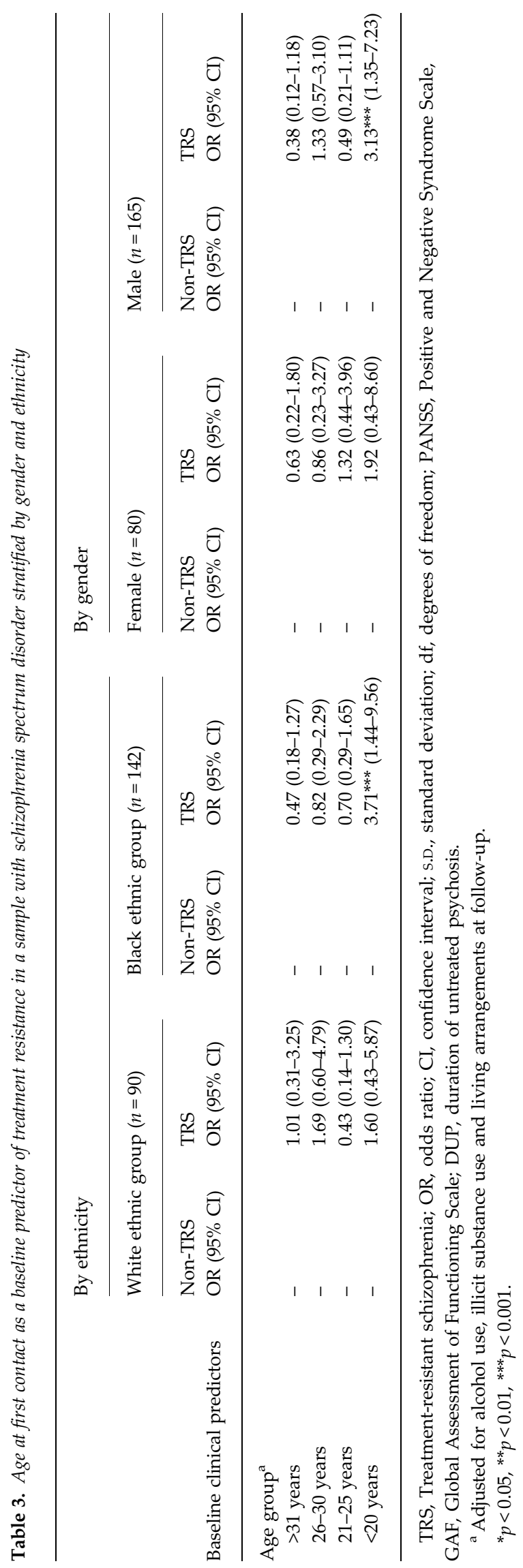


TR (Vanelle, 1995; Meltzer et al. 1997), although our study is the first longitudinal study in first-episode schizophrenia spectrum psychoses to identify this. These findings should alert clinicians to be more vigilant in individuals with a first episode of schizophrenia spectrum psychosis in late adolescence (age <20) in terms of their response (or lack thereof) to initial trials of antipsychotics and to be prepared to use clozapine more promptly when a lack of response is evident.

There was no difference between age of first contact in the TR group between males and females, although in the non-TR group, males were younger at the time of first contact. This observation, combined with the younger age of schizophrenia onset in those who eventually develop a TR course of illness is compatible with a greater role for neurodevelopmental impairment in this subgroup of schizophrenia patients, and a course of illness which differs premorbidly from that of nonTR patients. However, we did not find that the estimated deterioration in IQ scores, or a family history of psychosis was greater in the TR group compared to the non-TR group, and we did not identify that environmental risk factors were more prevalent in the non-TR group. These findings do not provide support for a neurodevelopmental pre-eminence in the TR group.

The severity of baseline clinical symptom profiles was not associated with TR over the course of the 5-year follow-up. Although, we were unable to identify any associations with increased negative symptomatology at first contact and the emergence of TR, they were predictive of those who had been treated with clozapine. Further, we found that increased severity of psychotic symptoms at baseline was associated with E-TR compared to those with non-TR.

\section{E-TR and L-TR}

Of the TR group, $70 \%$ displayed unremitting symptoms from the time of first antipsychotic treatment. The finding that $23 \%$ of the total population met the criteria for TR from illness onset indicates that this course of illness is not associated with prior antipsychotic use and raises the possibility that it may be a distinctive and homogenous schizophrenia subgroup, in line with evidence that there are biological differences between TR and treatment-responsive schizophrenia (Demjaha et al. 2012, 2014). This finding mirrors the rate of $20 \%$ who displayed 'chronicity' from illness outset in a FEP population (Wiersma et al. 1998), although is higher than a previous finding of $10 \%$ of FEP cases found to have shown no response to antipsychotics at the end of the first year of treatment (Robinson et al. 1999). Further, our finding that $70 \%$ of the TR group displayed E-TR, is similar to an earlier study in established schizophrenia, which identified that over half of patients with 'poor outcomes', remained psychotic from illness onset (Kolakowska et al. 1985). The high rates of early resistance of those with TR in this population is an important finding, which needs to be viewed in relation to the delay in clozapine use which exists in clinical practice (Howes et al. 2012), specifically the under use of clozapine within the first 1-2 years of illness presentation.

A minority of those with TR (i.e. 30\%) initially responded to antipsychotic medications, before developing L-TR. One possibility is that the loss of antipsychotic response may be due to the emergence of dopamine receptor supersensitivity in these cases. Dopamine supersensitivity is postulated to occur due to up-regulation of dopamine receptors and neural adaptation (Chouinard, 1991; Iyo et al. 2013) and it has been estimated that $50 \%$ of TR might be due to the emergence of dopamine supersensitivity (Chouinard \& Chouinard, 2008). Conversely, previous studies have found that over the course of illness, approximately one in six develop a lack of antipsychotic response subsequent to relapses (Wiersma et al. 1998; Emsley et al. 2012), a lower rate of L-TR than the 30\% identified in our study. It may be that there was a greater delay in treatment change for patients who relapsed in our naturalistic clinical setting, than in the closely monitored setting of the open label study of (Emsley et al. 2012) in which patients had a treatment initiation at the time of relapse. This delay in antipsychotic use, may have contributed to the higher rate of L-TR which we have identified, due to the evolving nature of TR in the context of relapse.

Our findings indicate that two distinct patterns of TR develops in patients, with the majority displaying TR from the onset, and a smaller subset of patients developing TR after periods of relapse, or with a later emergence of an intrinsic TR with a propensity for poorer outcomes and attenuated antipsychotic response overtime.

\section{Implications for practice}

Increasingly, cases of first-episode schizophrenia spectrum disorders in higher-income countries are managed within dedicated early intervention and FEP services; however, delays remain in the initiation of clozapine (Howes et al. 2012). In our study, 70\% of those with TR would have been most appropriately treated with clozapine at an early stage of their presentation, particularly given evidence that early treatment with clozapine is effective (Agid et al. 2011), and that worse outcomes are seen with a delayed use of clozapine (Nielsen et al. 2012a, Ucok et al. 2015). There is a need for a greater awareness and appreciation within 
FEP services that early resistance to antipsychotics is a not uncommon phenomenon. We would conjecture that on the basis of our findings consideration for early and judicious use of clozapine is appropriate.

\section{Access to clozapine}

Clozapine was commenced in less than half of those with TR over the course of the 5 years follow-up. Those who were commenced on clozapine in our study, were more likely to exhibit more severe psychotic symptoms, and increased negative symptomatology at baseline, when compared to those who 'met-criteria' for clozapine use, indicating that a more florid psychosis is likely a factor in a clinician's decision for the earlier use of clozapine in TR. Perhaps a more significant finding in relation to practice and clozapine initiation, was that those started on clozapine were significantly more likely to be living at home with family, compared to those who 'met criteria' for TR. Perhaps clozapine treatment is restricted for some who meet criteria for TR, due to a lack of family support (perceived or otherwise) or due to unstable living arrangements. These factors should prompt consideration for the provision of more dedicated hospital and community facilities to allow for the successful completion of the early stages of clozapine use, with adequate supported accommodation provided as required, to enable continued clozapine use. There is some evidence that such an approach is effective in increasing access to clozapine (Beck et al. 2014).

\section{Methodological considerations}

Strengths of this study include the quasi-prospective design which allowed for the follow up of first-episode schizophrenia spectrum psychosis cases over the first 5 years of illness. We have examined the course of TR in a naturalistic setting, from the time of the first use of antipsychotic medication, describing the pattern of TR onset, and identifying robust associations with the onset of TR.

Limitations to our study design include the small population of female patients and the lack of a robust measure of medication adherence, which may have affected the association with meeting the criteria for TR (McCutcheon et al. 2015). The unsystematic use of clozapine in this cohort is a further limitation in our estimation of TR, with over half of those meeting criteria for clozapine not treated with it.

We found notable clinical heterogeneity of course and outcome, with $34 \%$ of cases fulfilling the criteria for TR at 5 years, a relatively high rate, possibly reflecting the fact that a higher proportion were recruited from inpatient as opposed to community settings. A higher proportion recruited from inpatient settings, might indicate a greater clinical need, than those who were managed in the community setting (although who were not recruited to this study). However, we did not identify a greater illness severity as measured by total PANSS scores in those recruited from an inpatient setting compared to those recruited from a community setting. Nonetheless, the increased proportion of cases that were inpatients at the time of recruitment, and the recruitment from clinical services rather than by population screening or other methods may mean that the rate of TRS identified is an overestimate. We were unable to directly assess neurodevelopmental risk factors, (excepting a lack of difference in IQ and premorbid IQ between the TR and non-TR groups), which one might postulate would be increased in those with a higher risk of TR relating to a younger age of onset.

\section{Conclusions}

The findings from this study indicate that an early contact for psychosis during the ages of 18-20 years, particularly in males is a strong predictor of TRS. Seventy per cent of the TR subgroup presented with a TR picture at first contact, with no symptom remission occurring over the first 6 months of treatment. We did not find evidence suggesting that social factors and premorbid functioning were associated with the emergence of TR during the course of illness. Our findings reinforce the case for early assessment of TR in firstepisode patients so that clozapine may be considered and introduced promptly as a third-line treatment in first-episode schizophrenia. Future studies with larger samples are required to replicate and progress this important area of clinical prediction.

\section{Supplementary material}

The supplementary material for this article can be found at http://dx.doi.org/10.1017/S0033291716002014.

\section{Acknowledgements}

The study was funded by the UK National Institute of Health Research (NIHR) Specialist Biomedical Research Centre (BRC) for Mental Health, under its IMPACT Programme (Grant Reference Number RPPG-0606-1049), the NIHR BRC for Mental Health at SLaM NHS Foundation, and the IoPPN, King's College London (KCL), the Psychiatry Research Trust.

\section{Declaration of Interest}

S.S.S is supported by a European Research Council Consolidator Award. O.H. has received investigator- 
led grants and/or served as a speaker/consultant for Eli Lilly, Roche, Leyden-Delta, Lundbeck, Servier and Janssen-Cilag (J\&J). A.S.D. has received honoraria from Janssen and Roche Pharmaceuticals. F.G. has received honoraria for advisory work and lectures from Roche, BMS, Lundbeck, Otsaka and Sunovion, is a collaborator on a NHS Innovations project cofunded by Janssen and has a family member with professional links to Lilly and GSK, including share options. R.M.M. has received honoraria from Janssen, AstraZeneca, Lilly, BMS.

The other authors have no declaration of interest to make.

\section{References}

Agid O, Arenovich T, Sajeev G, Zipursky RB, Kapur S, Foussias G, Remington G (2011). An algorithm-based approach to first-episode schizophrenia: response rates over 3 prospective antipsychotic trials with a retrospective data analysis. Journal of Clinical Psychiatry 72, 1439-1444.

Andreasen NC, Carpenter Jr. WT, Kane JM, Lasser RA, Marder SR, Weinberger DR (2005). Remission in schizophrenia: proposed criteria and rationale for consensus. American Journal of Psychiatry 162, 441-449.

Beck K, McCutcheon R, Bloomfield MA, Gaughran F, Reis Marques T, MacCabe J, Selvaraj S, Taylor D, Howes OD (2014). The practical management of refractory schizophrenia - the Maudsley Treatment REview and Assessment Team service approach. Acta Psychiatrica Scandinavica 130, 427-438.

Carbon M, Correll CU (2014). Clinical predictors of therapeutic response to antipsychotics in schizophrenia. Dialogues in clinical neuroscience 16, 505-524.

Chouinard G (1991). Severe cases of neuroleptic-induced supersensitivity psychosis. Diagnostic criteria for the disorder and its treatment. Schizophrenia Research 5, 21-33.

Chouinard G, Chouinard VA (2008). Atypical antipsychotics: CATIE study, drug-induced movement disorder and resulting iatrogenic psychiatric-like symptoms, supersensitivity rebound psychosis and withdrawal discontinuation syndromes. Psychotherapy and Psychosomatics 77, 69-77.

Demjaha A, Egerton A, Murray RM, Kapur S, Howes OD, Stone JM, McGuire PK (2014). Antipsychotic treatment resistance in schizophrenia associated with elevated glutamate levels but normal dopamine function. Biological Psychiatry 75, e11-e13.

Demjaha A, Murray RM, McGuire PK, Kapur S, Howes OD (2012). Dopamine synthesis capacity in patients with treatment-resistant schizophrenia. American Journal of Psychiatry 169, 1203-1210.

Di Forti M, Morgan C, Dazzan P, Pariante C, Mondelli V, Marques TR, Handley R, Luzi S, Russo M, Paparelli A, Butt A, Stilo SA, Wiffen B, Powell J, Murray RM (2009). High-potency cannabis and the risk of psychosis. British Journal of Psychiatry 195, 488-491.
Di Forti M, Sallis H, Allegri F, Trotta A, Ferraro L, Stilo SA, Marconi A, La Cascia C, Reis Marques T, Pariante C, Dazzan P, Mondelli V, Paparelli A, Kolliakou A, Prata D, Gaugrhan F, David AS, Morgan C, Sthal D, Khondoker M, MacCabe JH, Murray RM (2014). Daily use, especially of high-potency cannabis, drives the earlier onset of psychosis in cannabis users. Schizophrenia Bulletin 40, 1509-1517.

Emsley R, Nuamah I, Hough D, Gopal S (2012). Treatment response after relapse in a placebo-controlled maintenance trial in schizophrenia. Schizophrenia Research 138, 29-34.

Frank J, Lang M, Witt SH, Strohmaier J, Rujescu D, Cichon S, Degenhardt F, Nothen MM, Collier DA, Ripke S, Naber D, Rietschel M (2015). Identification of increased genetic risk scores for schizophrenia in treatment-resistant patients. Molecular Psychiatry 20, 150-151.

Heinze G, Schemper M (2002). A solution to the problem of separation in logistic regression. Statistics in Medicine 21, 2409-2419.

Howes OD, Vergunst F, Gee S, McGuire P, Kapur S, Taylor D (2012). Adherence to treatment guidelines in clinical practice: study of antipsychotic treatment prior to clozapine initiation. British Journal of Psychiatry 201, 481-485.

Iyo M, Tadokoro S, Kanahara N, Hashimoto T, Niitsu T, Watanabe H, Hashimoto K (2013). Optimal extent of dopamine D2 receptor occupancy by antipsychotics for treatment of dopamine supersensitivity psychosis and late-onset psychosis. Journal of Clinical Psychopharmacology 33, 398-404.

Kay SR, Fiszbein A, Opler LA (1987). The positive and negative syndrome scale (PANSS) for schizophrenia. Schizophrenia Bulletin 13, 261-276.

Kolakowska T, Williams AO, Ardern M, Reveley MA, Jambor K, Gelder MG, Mandelbrote BM (1985). Schizophrenia with good and poor outcome. I: Early clinical features, response to neuroleptics and signs of organic dysfunction. British Journal of Psychiatry 146, 229-239.

Leucht S, Samara M, Heres S, Patel MX, Woods SW, Davis JM (2014). Dose equivalents for second-generation antipsychotics: the minimum effective dose method. Schizophrenia Bulletin 40, 314-326.

Lewis SW, Barnes TR, Davies L, Murray RM, Dunn G, Hayhurst KP, Markwick A, Lloyd H, Jones PB (2006). Randomized controlled trial of effect of prescription of clozapine versus other second-generation antipsychotic drugs in resistant schizophrenia. Schizophrenia Bulletin 32, 715-723.

Lin CC, Wang YC, Chen JY, Liou YJ, Bai YM, Lai IC, Chen TT, Chiu HW, Li YC (2008). Artificial neural network prediction of clozapine response with combined pharmacogenetic and clinical data. Computer Methods and Programs in Biomedicine 91, 91-99.

Malla A, Norman R, Schmitz N, Manchanda R, BechardEvans L, Takhar J, Haricharan R (2006). Predictors of rate and time to remission in first-episode psychosis: a two-year outcome study. Psychological Medicine 36, 649-658.

Mallett R, Leff J, Bhugra D, Pang D, Zhao JH (2002). Social environment, ethnicity and schizophrenia. A case-control study. Social Psychiatry and Psychiatric Epidemiology 37, 329-335.

Martin AK, Mowry B (2016). Increased rare duplication burden genomewide in patients with treatment-resistant schizophrenia. Psychological Medicine 46, 469-476. 
McCutcheon R, Beck K, Bloomfield MA, Marques TR, Rogdaki M, Howes OD (2015). Treatment resistant or resistant to treatment? Antipsychotic plasma levels in patients with poorly controlled psychotic symptoms. Journal of Psychopharmacology 29, 892-897.

McGuffin P, Farmer A, Harvey I (1991). A polydiagnostic application of operational criteria in studies of psychotic illness. Development and reliability of the OPCRIT system. Archives of General Psychiatry 48, 764-770.

Meltzer HY (1997). Treatment-resistant schizophrenia-the role of clozapine. Current Medical Research and Opinion 14, $1-20$.

Meltzer HY, Rabinowitz J, Lee MA, Cola PA, Ranjan R, Findling RL, Thompson PA (1997). Age at onset and gender of schizophrenic patients in relation to neuroleptic resistance. American Journal of Psychiatry 154, 475-482.

Menezes NM, Arenovich T, Zipursky RB (2006). A systematic review of longitudinal outcome studies of firstepisode psychosis. Psychological Medicine 36, 1349-1362.

Morgan C, Lappin J, Heslin M, Donoghue K, Lomas B, Reininghaus $\mathrm{U}$, Onyejiaka A, Croudace T, Jones PB, Murray RM, Fearon P, Doody GA, Dazzan P (2014). Reappraising the long-term course and outcome of psychotic disorders: the AESOP-10 study. Psychological Medicine 44, 2713-2726.

NICE (2014). Psychosis and Schizophrenia in Adults: Treatment and Management (Clinical Guideline 178). Royal College of Psychiatrists: London.

Nielsen J, Nielsen RE, Correll CU (2012a). Predictors of clozapine response in patients with treatment-refractory schizophrenia: results from a Danish Register Study. Journal of Clinical Psychopharmacology 32, 678-683.

Nielsen J, Roge R, Schjerning O, Sorensen HJ, Taylor D (2012b). Geographical and temporal variations in clozapine prescription for schizophrenia. European Neuropsychopharmacology 22, 818-824.

Ortiz BB, Araujo Filho GM, Araripe Neto AG, Medeiros D, Bressan RA (2013). Is disorganized schizophrenia a predictor of treatment resistance? Evidence from an observational study. Revista Brasileira de Psiquiatria 35, 432-434.

Revier CJ, Reininghaus U, Dutta R, Fearon P, Murray RM, Doody GA, Croudace T, Dazzan P, Heslin M, Onyejiaka A, Kravariti E, Lappin J, Lomas B, Kirkbride JB, Donoghue K, Morgan C, Jones PB (2015). Ten-year outcomes of first-episode psychoses in the MRC AESOP-10 study. Journal of Nervous and Mental Disease 203, 379-386.

Robinson DG, Woerner MG, Alvir JM, Geisler S, Koreen A, Sheitman B, Chakos M, Mayerhoff D, Bilder R, Goldman R, Lieberman JA (1999). Predictors of treatment response from a first episode of schizophrenia or schizoaffective disorder. American Journal of Psychiatry 156, 544-549.

Ropcke B, Eggers C (2005). Early-onset schizophrenia: a 15year follow-up. European Child \& Adolescent Psychiatry 14, 341-350.

Sartorius N, Gulbinat W, Harrison G, Laska E, Siegel C (1996). Long-term follow-up of schizophrenia in 16 countries. A description of the International Study of
Schizophrenia conducted by the World Health Organization. Social Psychiatry and Psychiatric Epidemiology 31, 249-258.

Schennach R, Riedel M, Musil R, Moller HJ (2012). Treatment Response in First-episode Schizophrenia. Clinical Psychopharmacology and Neuroscience 10, 78-87.

Schneider C, Papachristou E, Wimberley T, Gasse C, Dima D, MacCabe JH, Mortensen PB, Frangou S (2015).

Clozapine use in childhood and adolescent schizophrenia: A nationwide population-based study. European Neuropsychopharmacology 25, 857-863.

Singh SP, Cooper JE, Fisher HL, Tarrant CJ, Lloyd T, Banjo J, Corfe S, Jones P (2005). Determining the chronology and components of psychosis onset: The Nottingham Onset Schedule (NOS). Schizophrenia Research 80, 117-130.

Stroup TS, Gerhard T, Crystal S, Huang C, Olfson M (2014). Geographic and clinical variation in clozapine use in the United States. Psychiatric Services 65, 186-192.

Stroup TS, Gerhard T, Crystal S, Huang C, Olfson M (2016). Comparative effectiveness of clozapine and standard antipsychotic treatment in adults with schizophrenia. American Journal of Psychiatry 173, 166-173.

Ucok A, Cikrikcili U, Karabulut S, Salaj A, Ozturk M, Tabak O, Durak R (2015). Delayed initiation of clozapine may be related to poor response in treatment-resistant schizophrenia. International Clinical Psychopharmacology 30, 290-295.

Vanelle JM (1995). Treatment refractory schizophrenia [in French]. L'Encephale 21 (Spec. No. 3), 13-21.

Vanelle JM, Olie JP, Levy-Soussan P (1994). New antipsychotics in schizophrenia: the French experience. Acta Psychiatrica Scandinavica (Suppl.) 380, 59-63.

Vyas NS, Hadjulis M, Vourdas A, Byrne P, Frangou S (2007). The Maudsley early onset schizophrenia study. Predictors of psychosocial outcome at 4-year follow-up. European Child \& Adolescent Psychiatry 16, 465-470.

Wechsler D (1997). Wechsler Adult Intelligence Scale. The Psychological Corporation: San Antonio, TX.

Wechsler D (2001). Wechsler Test of Adult Reading: WTAR. Psychological Corporation: San Antonio, TX.

Wheeler A, Humberstone V, Robinson G (2009). Outcomes for schizophrenia patients with clozapine treatment: how good does it get? Journal of Psychopharmacology 23, 957-965.

WHO (1992). The ICD-10 Classification of Mental and Behavioural Disorders: Clinical Descriptions and Diagnostic Guidelines. World Health Organization: Geneva.

WHO (1994). Schedules for Clinical Assessment in Neuropsychiatry: Version 2: Manual. World Health Organization, Division of Mental Health: Geneva.

Wiersma D, Nienhuis FJ, Slooff CJ, Giel R (1998). Natural course of schizophrenic disorders: a 15-year followup of a Dutch incidence cohort. Schizophrenia Bulletin 24, 7585.

Wimberley T, Støvring H, Sørensen HJ, Horsdal HT, MacCabe JH, Gasse C (2016). Predictors of treatment resistance in patients with schizophrenia: a populationbased cohort study. Lancet Psychiatry 3, 358-366. 